\title{
INTEGRAL EQUATIONS OF THE FIRST KIND IN PLANE ELASTICITY
}

\author{
BY \\ CHRISTIAN CONSTANDA \\ Department of Mathematics, University of Strathclyde, Glasgow, Scotland, U.K.
}

\begin{abstract}
A modified single layer potential is used to solve the interior and exterior Dirichlet problems of plane strain by means of integral equations of the first kind for all smooth boundary curves, including those on which the classical method fails to operate.
\end{abstract}

Introduction. It is well known [1] that the so-called direct method for the Dirichlet problem in plane elasticity reduces to the solution of an integral equation of the first kind for the unknown density of a single layer potential. However, there are boundary curves on which this procedure cannot be applied, since the corresponding homogeneous equation has nonzero solutions [2]. To circumvent this obstacle, in [3] the solution of the exterior Dirichlet problem is sought as the sum of a classical single layer potential and a rigid displacement, under the assumption that either the rotation element of the rigid displacement or the total moment on the boundary is known. In this paper we propose to obtain the solution in the form of a modified single layer potential on any smooth boundary, without prescribing additional data. We specify fully the arbitrariness of the representation and indicate how the solution of a very general exterior Dirichlet problem can be obtained from that of the interior one with the same boundary values.

Prerequisites. In what follows, $\mathscr{M}_{p \times q}$ is the space of $p \times q$ matrices, $H^{(i)}$ are the columns of a matrix $H \in \mathscr{M}_{p \times q}, E_{n}$ is the identity element in $\mathscr{M}_{n \times n}$, and a superscript $\mathrm{T}$ denotes matrix transposition. To simplify the notation, if $X$ is a space of scalar functions and $\varphi \in \mathscr{M}_{p \times q}$, then $\varphi \in X$ means that every component of $\varphi$ belongs to $X$. Also, if $\mathscr{L}$ is an operator defined on functions $\theta \in \mathscr{M}_{p \times 1}$ and such that $\mathscr{L} \theta \in \mathscr{M}_{r \times 1}$, and $\Theta \in \mathscr{M}_{p \times q}$, then $\mathscr{L} \Theta \in \mathscr{M}_{r \times q}$ is the matrix with $(\mathscr{L} \boldsymbol{\Theta})^{(i)}=\mathscr{L} \boldsymbol{\Theta}^{(i)}$.

Let $S^{+}$be a finite domain in $\mathbb{R}^{2}$ bounded by a closed $C^{2}$-curve $\partial S$, and let $S^{-}=\mathbb{R}^{2} \backslash S^{+} \cup \partial S$. The equilibrium equations of plane strain in the absence of

Received June 28, 1993.

1991 Mathematics Subject Classification. Primary 45B05, 73C35.

The author would like to acknowledge the financial support received from the Carnegie Trust for the Universities of Scotland during the progress of this work.

(C)1995 Brown University 
body forces can be written in the form

$$
A\left(\partial_{x}\right) u=0,
$$

where $u=\left(u_{1}, u_{2}\right)^{\mathrm{T}}$ is the displacement vector, $A\left(\partial_{x}\right)=A\left(\partial / \partial x_{1}, \partial / \partial x_{2}\right)$,

$$
A\left(\xi_{1}, \xi_{2}\right)=\left(\begin{array}{cc}
\mu \Delta+(\lambda+\mu) \xi_{1}^{2} & (\lambda+\mu) \xi_{1} \xi_{2} \\
(\lambda+\mu) \xi_{1} \xi_{2} & \mu \Delta+(\lambda+\mu) \xi_{2}^{2}
\end{array}\right),
$$

$\Delta=\xi_{1}^{2}+\xi_{2}^{2}$, and $\lambda$ and $\mu$ are the Lamé constants of the (homogeneous and isotropic) material occupying $S^{+}$or $S^{-}$. We assume that $\lambda+\mu>0$ and $\mu>0$, so that (1) is an elliptic system.

We also consider the boundary stress operator $T\left(\partial_{x}\right)=T\left(\partial / \partial x_{1}, \partial / \partial x_{2}\right)$ defined by

$$
T\left(\xi_{1}, \xi_{2}\right)=\left(\begin{array}{cc}
(\lambda+2 \mu) \nu_{1} \xi_{1}+\mu \nu_{2} \xi_{2} & \mu \nu_{2} \xi_{1}+\lambda \nu_{1} \xi_{2} \\
\lambda \nu_{2} \xi_{1}+\mu \nu_{1} \xi_{2} & \mu \nu_{1} \xi_{1}+(\lambda+2 \mu) \nu_{2} \xi_{2}
\end{array}\right),
$$

where $\nu=\left(\nu_{1}, \nu_{2}\right)^{\mathrm{T}}$ is the unit outward normal to $\partial S$. It can be shown by direct calculation that

$$
\int_{S^{+}} F^{\mathrm{T}} A u d \sigma=\int_{\partial S} F^{\mathrm{T}} T u d s,
$$

where the columns $F^{(i)}$ of the matrix

$$
F=\left(\begin{array}{rrr}
1 & 0 & x_{2} \\
0 & 1 & -x_{1}
\end{array}\right)
$$

form a basis for the space of rigid displacements. Evidently, $A F=0$ in $\mathbb{R}^{2}, T F=0$ on $\partial S$, and a general rigid displacement can be written as $F k$, with a constant $k \in \mathscr{M}_{3 \times 1}$.

A matrix of fundamental solutions for the operator $A$ is [4]

$$
D(x, y)=A^{*}\left(\partial_{x}\right) t(x, y),
$$

where $A^{*}$ is the adjoint of $A$ and

$$
t(x, y)=-[8 \pi \mu(\lambda+2 \mu)]^{-1}|x-y|^{2} \ln |x-y| .
$$

We introduce the single layer potential

$$
(V \varphi)(x)=\int_{\partial S} D(x, y) \varphi(y) d s(y),
$$

and the double layer potential

$$
(W \varphi)(x)=\int_{\partial S} P(x, y) \varphi(y) d s(y),
$$

where $\varphi \in \mathscr{M}_{2 \times 1}$ is the density and

$$
P(x, y)=\left[T\left(\partial_{y}\right) D(y, x)\right]^{\mathrm{T}} .
$$


Theorem 1. (i) If $\varphi \in C(\partial S)$, then $V \varphi$ and $W \varphi$ are analytic in $S^{+} \cup S^{-}$and

$$
A(V \varphi)=A(W \varphi)=0 \text { in } S^{+} \cup S^{-} .
$$

(ii) If $\varphi \in C^{0, \alpha}(\partial S), \alpha \in(0,1)$, then the direct values $V_{0} \varphi$ and $W_{0} \varphi$ of $V \varphi$ and $W \varphi$ on $\partial S$ exist (the latter in the sense of principal value), the functions

$$
\mathscr{V}^{+}(\varphi)=\left.(V \varphi)\right|_{\bar{S}^{+}}, \quad \mathscr{V}^{-}(\varphi)=\left.(V \varphi)\right|_{\bar{S}^{-}}
$$

are of class $C^{1, \alpha}\left(\bar{S}^{+}\right)$and $C^{1, \alpha}\left(\bar{S}^{-}\right)$, respectively, and

$$
T \mathscr{V}^{+}(\varphi)=\left(W_{0}^{*}+\frac{1}{2} I\right) \varphi, \quad T \mathscr{V}^{-}(\varphi)=\left(W_{0}^{*}-\frac{1}{2} I\right) \varphi,
$$

where $W_{0}^{*}$ is the adjoint of $W_{0}$ and $I$ is the identity operator.

(iii) If $\varphi \in C^{1, \alpha}(\partial S), \alpha \in(0,1)$, then the functions

$$
\mathscr{W}^{+}(\varphi)=\left\{\begin{array}{ll}
\left.(W \varphi)\right|_{S^{+}} & \text {in } S^{+}, \\
\left(W_{0}-\frac{1}{2} I\right) \varphi & \text { on } \partial S,
\end{array} \quad \mathscr{W}^{-}(\varphi)= \begin{cases}\left.(W \varphi)\right|_{S^{-}} & \text {in } S^{-}, \\
\left(W_{0}+\frac{1}{2} I\right) \varphi & \text { on } \partial S\end{cases}\right.
$$

are of class $C^{1, \alpha}\left(\bar{S}^{+}\right)$and $C^{1, \alpha}\left(\bar{S}^{-}\right)$, respectively.

(iv) $\left(W_{0}-\frac{1}{2} I\right) \varphi=0$ if and only if $\varphi=0 ;\left(W_{0}+\frac{1}{2} I\right) \varphi=0$ if and only if $\varphi$ is a rigid displacement $F k$.

(v) $\left(W_{0}^{*}-\frac{1}{2} I\right) \varphi=0$ if and only if $\varphi=0$; the solutions of $\left(W_{0}^{*}+\frac{1}{2} I\right) \varphi=0$ form a three-dimensional subspace of $C^{1, \alpha}(\partial S)$.

(vi) Let $\mathscr{A}$ be the class of functions $u \in \mathscr{M}_{2 \times 1}$ that, as $r=|x| \rightarrow \infty$, admit an asymptotic expansion of the form

$$
\begin{aligned}
& u_{1}(r, \theta)=r^{-1}\left(\alpha m_{0} \sin \theta+m_{1} \cos \theta+m_{0} \sin 3 \theta+m_{2} \cos 3 \theta\right)+O\left(r^{-2}\right), \\
& u_{2}(r, \theta)=r^{-1}\left(m_{3} \sin \theta+\alpha m_{0} \cos \theta+m_{4} \sin 3 \theta-m_{0} \cos 3 \theta\right)+O\left(r^{-2}\right),
\end{aligned}
$$

where $m_{0}, \ldots, m_{4}$ are arbitrary constants and $\alpha=(\lambda+3 \mu) /(\lambda+\mu)$. Also, let $\mathscr{A}^{*}$ be the class of functions of the form $u=F k+\sigma^{\mathscr{A}}$, with $\sigma^{\mathscr{A}} \in \mathscr{A}$. Then

$$
V \varphi=M^{\infty}(p \varphi)+\sigma^{\mathscr{A}}
$$

where $p$ is the operator defined on continuous functions $\varphi \in \mathscr{M}_{2 \times 1}$ on $\partial S$ by

$$
p \varphi=\int_{\partial S} F^{\mathrm{T}} \varphi d s
$$

and

$$
\begin{aligned}
& 4 \pi \mu(\alpha+1) M^{\infty}(r, \theta) \\
& \quad=\left(\begin{array}{ccr}
-2 \alpha(\ln r+1)+\cos 2 \theta & \sin 2 \theta & r^{-1}(\alpha+1) \sin \theta \\
\sin 2 \theta & -2 \alpha(\ln r+1)-\cos 2 \theta & -r^{-1}(\alpha+1) \cos \theta
\end{array}\right),
\end{aligned}
$$

with $A M^{\infty}=0$ in $\mathbb{R}^{2}$.

(vii) (Somigliana formulae.) If $u \in C^{2}\left(S^{+}\right) \cap C^{1}\left(\bar{S}^{+}\right)$is a solution of (1) in $S^{+}$, then

$$
V\left(\left.(T u)\right|_{\partial S}\right)-W\left(\left.u\right|_{\partial S}\right)=\left\{\begin{aligned}
u & \text { in } S^{+} \\
\frac{1}{2} u & \text { on } \partial S \\
0 & \text { in } S^{-}
\end{aligned}\right.
$$


(viii) The interior Dirichlet problem has at most one solution $u \in C^{2}\left(S^{+}\right) \cap$ $C^{1}\left(\bar{S}^{+}\right)$. The exterior Dirichlet problem has at most one solution $u \in C^{2}\left(S^{-}\right) \cap$ $C^{1}\left(\bar{S}^{-}\right) \cap \mathscr{A}^{*}$.

(ix) For any closed $C^{2}$-curve $\partial S$, there are a unique $\Phi \in \mathscr{M}_{2 \times 3}$ and a unique constant $\mathscr{C} \in \mathscr{M}_{3 \times 3}$ such that $\left\{\Phi^{(i)}\right\}$ is a basis for the null space of $W_{0}^{*}+\frac{1}{2} I$ (see $(\mathrm{v}))$ and

$$
V_{0} \Phi=F \mathscr{C}, \quad p \Phi=E_{3} .
$$

A discussion of (i)-(viii) can be found in [4]. The proof of (ix) is given in [2].

Instead of $D(x, y)$ we consider the matrix of fundamental solutions $D^{H}(x, y)$ given by

$$
D^{H}(x, y)=D(x, y)+F(x) H F^{\mathrm{T}}(y),
$$

where $H \in \mathscr{M}_{3 \times 3}$ is a constant symmetric matrix. Using $D^{H}$, we construct the modified single layer potential

$$
\left(V^{H} \varphi\right)(x)=\int_{\partial S} D^{H}(x, y) \varphi(y) d s(y)=(V \varphi)(x)+F H p \varphi .
$$

It is clear from (3) and (4) that the modified double layer potential coincides with $W$.

Theorem 2. If $\operatorname{det}(H+\mathscr{C}) \neq 0$ and $V_{0}^{H} \varphi=0$ on $\partial S$, then $\varphi=0$.

Proof. By (5) and Theorem 1(i, ix), the function $U=V^{H} \varphi-(V \Phi) p \varphi+F \mathscr{C} p \varphi$ satisfies

$$
\begin{aligned}
A U & =0 \text { in } S^{-}, \\
U & =0 \text { on } \partial S, \\
U & =V(\varphi-\Phi p \varphi)+F(H+\mathscr{C}) p \varphi \text { as }|x| \rightarrow \infty .
\end{aligned}
$$

Since, by Theorem 1(ix), $p(\varphi-\Phi p \varphi)=0$, from Theorem 1(vi) it follows that

$$
U=F(H+\mathscr{C}) p \varphi+\sigma^{\mathscr{A}} \text { as }|x| \rightarrow \infty .
$$

Applying Theorem 1(viii), we conclude that $U=0$ in $S^{-}$; consequently, $F(H+\mathscr{C}) p \varphi=0$. Then the linear independence of the $F^{(i)}$ yields $(H+\mathscr{C}) p \varphi=0$, which, in view of the assumption that $\operatorname{det}(H+\mathscr{C}) \neq 0$, leads to $p \varphi=0$. Hence, $V^{H} \varphi=V \varphi$, and

$$
\begin{aligned}
A(V \varphi) & =0 \text { in } S^{+} \cup S^{-}, \\
V_{0} \varphi & =0 \text { on } \partial S, \\
V \varphi & \in \mathscr{A} .
\end{aligned}
$$

By Theorem 1(viii), $V \varphi=0$ in $\mathbb{R}^{2}$. Using Theorem 1(ii), we now deduce that $\varphi=0$.

Composition of boundary operators. By Theorem 1 (ii), for arbitrary $\varphi \in C^{0, \alpha}(\partial S)$

$$
\left.\mathscr{V}^{+}(\varphi)\right|_{\partial S}=\theta \in C^{1, \alpha}(\partial S),\left.\quad T \mathscr{V}^{+}(\varphi)\right|_{\partial S}=\chi \in C^{0, \alpha}(\partial S)
$$


Since $A \mathscr{V}^{+}(\varphi)=0$ in $S^{+}$, from the Somigliana relations (Theorem 1(vii)) it follows that

or

$$
\begin{aligned}
\mathscr{V}^{+}(\varphi) & =V \chi-W \theta \text { in } S^{+}, \\
\frac{1}{2} \theta & =V_{0} \chi-W_{0} \theta \text { on } \partial S,
\end{aligned}
$$

$$
\mathscr{V}^{+}(\varphi)=\mathscr{V}^{+}(\chi)-\mathscr{W}^{+}(\theta) \text { in } \bar{S}^{+} .
$$

We introduce an operator $N_{0}: C^{1, \alpha}(\partial S) \rightarrow C^{0, \alpha}(\partial S)$ by

$$
N_{0} \theta=T \mathscr{W}^{+}(\theta) \text {. }
$$

Then from (7)

$$
T \mathscr{V}^{+}(\varphi)=T \mathscr{V}^{+}(\chi)-N_{0} \theta \text { on } \partial S,
$$

which, in view of Theorem 1 (ii), can be written as

$$
\frac{1}{2} \chi=W_{0}^{*} \chi-N_{0} \theta \text {. }
$$

Also, the equality $\left.T \mathscr{V}^{+}(\varphi)\right|_{\partial S}=\chi$ and Theorem 1(ii) yield

$$
\chi=\frac{1}{2} \varphi+W_{0}^{*} \varphi .
$$

Substituting $\theta=\left.\mathscr{V}^{+}(\varphi)\right|_{\partial S}=V_{0} \varphi$ and $\chi$ from (10) in (6) and (9), and taking the arbitrariness of $\varphi \in C^{0, x}(\partial S)$ into account, we find that

$$
W_{0} V_{0}=V_{0} W_{0}^{*}, \quad N_{0} V_{0}=W_{0}^{* 2}-\frac{1}{4} I \quad \text { on } \quad C^{0, \alpha}(\partial S) .
$$

Now for arbitrary $\psi \in C^{1, \alpha}(\partial S)$, by Theorem 1(iii),

$$
\left.\mathscr{W}^{+}(\psi)\right|_{\partial S}=\zeta \in C^{1, \alpha}(\partial S),\left.\quad T \mathscr{W}^{+}(\psi)\right|_{\partial S}=\eta \in C^{0, \alpha}(\partial S) .
$$

Given that $A \mathscr{W}^{+}(\psi)=0$ in $S^{+}$, the Somigliana relations show that

$$
\begin{aligned}
\mathscr{W}^{+}(\psi) & =V \eta-W \zeta \text { in } S^{+}, \\
\frac{1}{2} \zeta & =V_{0} \eta-W_{0} \zeta \text { on } \partial S,
\end{aligned}
$$

or

$$
\mathscr{W}^{+}(\psi)=\mathscr{V}^{+}(\eta)-\mathscr{W}^{+}(\zeta) \text { in } \bar{S}^{+} .
$$

Applying $T$ to this equality, from Theorem 1(ii, iii) we see that

$$
\frac{1}{2} \eta=W_{0}^{*} \eta-N_{0} \zeta
$$

At the same time, by $(8),\left.T \mathscr{W}^{+}(\psi)\right|_{j S}=\eta$ can be written as

$$
\eta=N_{0} \psi \text {. }
$$

Replacing $\zeta=\left.\mathscr{W}^{+}(\psi)\right|_{\partial S}=-\frac{1}{2} \psi+W_{0} \psi$ (see Theorem 1(iii)) and $\eta$ from (14) in (12) and (13), and recalling that $\psi \in C^{1, \alpha}(\partial S)$ is arbitrary, we find that

$$
N_{0} W_{0}=W_{0}^{*} N_{0}, \quad V_{0} N_{0}=W_{0}^{2}-\frac{1}{4} I \quad \text { on } C^{1, \alpha}(\partial S) .
$$

THEOREM 3. The composition formulae (11) and (15) remain valid for the modified potential $V^{H}$. 
Proof. Since $V_{0}^{H}=V_{0}+F H p$, we only need to show that

$$
\begin{aligned}
& W_{0}(F H p)=F H p W_{0}^{*}, \quad N_{0}(F H p)=0 \text { on } C^{0, \alpha}(\partial S), \\
& p N_{0}=0 \text { on } C^{1, \alpha}(\partial S) .
\end{aligned}
$$

(i) For any $\varphi \in C^{0, \alpha}(\partial S)$

$$
\begin{aligned}
\left(p W_{0}^{*}\right) \varphi & =p\left(W_{0}^{*} \varphi\right)=\int_{\partial S} F^{\mathrm{T}} W_{0}^{*} \varphi d s \\
& =\int_{\partial S} F^{\mathrm{T}}(x)\left[\int_{\partial S} T\left(\partial_{x}\right) D(x, y) \varphi(y) d s(y)\right] d s(x) \\
& =\int\left[\int_{\partial S} F^{\mathrm{T}}(x) T\left(\partial_{x}\right) D(x, y) d s(x)\right] \varphi(y) d s(y) .
\end{aligned}
$$

From the Somigliana formula for $F$ we find that for $x \in \partial S$

$$
\frac{1}{2} F(x)=-\int_{\partial S}\left[T\left(\partial_{y}\right) D(y, x)\right]^{\mathrm{T}} F(y) d s(y)=-\left[\int_{\partial S} F^{\mathrm{T}}(y) T\left(\partial_{y}\right) D(y, x) d s(y)\right]^{\mathrm{T}} ;
$$

therefore,

$$
\left(p W_{0}^{*}\right) \varphi=-\frac{1}{2} \int_{\partial S} F^{\mathrm{T}} \varphi d s=-\frac{1}{2} p \varphi,
$$

from which $F H\left(p W_{0}^{*}\right) \varphi=-\frac{1}{2} F H p \varphi$. Using the Somigliana formula for $F$ on $\partial S$ again, this time in the form $\frac{1}{2} F=-W_{0} F$, we obtain

$$
F H\left(p W_{0}^{*}\right) \varphi=W_{0}(F H p) \varphi,
$$

which proves the first assertion.

(ii) Since $p \varphi \in \mathscr{M}_{3 \times 1}$ is constant, for any $\varphi \in C^{0, \alpha}(\partial S)$

$$
N_{0}(F H p) \varphi=N_{0}(F H p \varphi)=N_{0}(F H)(p \varphi)=T \mathscr{W}^{+}(F H)(p \varphi) .
$$

From the Somigliana relations for $F$ we see that

$$
\mathscr{W}^{+}(F H)=-F H \text { in } \bar{S}^{+} \text {; }
$$

consequently,

$$
N_{0}(F H p) \varphi=-(T F H) p \varphi=0 .
$$

(iii) By (2), for any $\varphi \in C^{1, \alpha x}(\partial S)$

$$
\begin{aligned}
\left(p N_{0}\right) \varphi & =p\left(N_{0} \varphi\right)=\int_{\partial S} F^{\mathrm{T}} N_{0} \varphi d s=\int_{\partial S} F^{\mathrm{T}} T \mathscr{W}^{+}(\varphi) d s \\
& =\int_{S^{+}} F^{\mathrm{T}} A \mathscr{W}^{+}(\varphi) d \sigma=\int_{S^{+}} F^{\mathrm{T}} A(W \varphi) d \sigma=0 .
\end{aligned}
$$

THEOREM 4. (i) $\frac{1}{4}$ is an eigenvalue of $W_{0}^{2}$ and $W_{0}^{* 2}$, and the corresponding eigenspaces are the same as those of $W_{0}$ and $W_{0}^{*}$, respectively, for the eigenvalue $-\frac{1}{2}$. 
(ii) If $N_{0} \varphi=0$, then $\varphi$ is a rigid displacement.

Proof. (i) We can write

$$
\left(W_{0}^{2}-\frac{1}{4} I\right) \varphi=\left(W_{0}-\frac{1}{2} I\right)\left(\left(W_{0}+\frac{1}{2} I\right) \varphi\right)=0 .
$$

Since, by Theorem 1 (iv), $\frac{1}{2}$ is not an eigenvalue of $W_{0}$, it follows that $\left(W_{0}+\frac{1}{2} I\right) \varphi=$ 0 . We now apply Theorem 1 (iv) with respect to $-\frac{1}{2}$.

The proof for $W_{0}^{* 2}$ is similar.

(ii) By (15),

$$
0=V_{0}\left(N_{0} \varphi\right)=\left(V_{0} N_{0}\right) \varphi=\left(W_{0}^{2}-\frac{1}{4} I\right) \varphi,
$$

and the result follows from (i).

The interior Dirichlet problem. We want to find $u \in C^{2}\left(S^{+}\right) \cap C^{1}\left(\bar{S}^{+}\right)$such that

$$
\begin{aligned}
A u & =0 \text { in } S^{+}, \\
u & =f \text { on } \partial S .
\end{aligned}
$$

We seek the solution in the form $u=V^{H} \varphi$, with $H$ chosen so that $\operatorname{det}(H+\mathscr{C}) \neq$ 0 . Then, by Theorem 1(ii), the problem reduces to solving the first-kind equation

$$
V_{0}^{H} \varphi=f .
$$

Theorem 5. Equation (17) has a unique $C^{0, \alpha}$-solution if and only if $f \in C^{1, \alpha}(\partial S)$. In this case, the solution $\varphi$ also satisfies

$$
\left(W_{0}^{* 2}-\frac{1}{4} I\right) \varphi=N_{0} f .
$$

Proof. If $\varphi \in C^{0, r}(\partial S)$ is the solution of (17), then, by Theorem 1(ii), $f \in$ $C^{1, \alpha}(\partial S)$. By Theorem 3 and (11), we also have

$$
\left(W_{0}^{* 2}-\frac{1}{4} I\right) \varphi=\left(N_{0} V_{0}^{H}\right) \varphi=N_{0}\left(V_{0}^{H} \varphi\right)=N_{0} f .
$$

Now let $f \in C^{1, \alpha}(\partial S)$. Then $N_{0} f \in C^{0, \alpha}(\partial S)$. Since $\left\{F^{(i)}\right\}$ is a basis for the null space of $W_{0}^{2}-\frac{1}{4} I$ and since, by (16) with $\varphi$ replaced by $f, \int_{\partial S} F^{\mathrm{T}} N_{0} f d s=0$, it follows that $(18)$ is soluble. We write its general solution in the form

$$
\varphi=\varphi_{0}+\Phi a,
$$

where $\varphi_{0}$ is a specific ("fixed") solution of (18), $\left\{\Phi^{(i)}\right\}$ is a basis for the null space of $W_{0}^{* 2}-\frac{1}{4} I$ (see Theorems $4(\mathrm{i})$ and $1(\mathrm{v})$ ) and $a \in \mathscr{M}_{3 \times 1}$ is a constant vector. Then, by Theorem 3 and (11),

$$
\begin{aligned}
0 & =\left(W_{0}^{* 2}-\frac{1}{4} I\right)\left(\varphi_{0}+\Phi a\right)-N_{0} f \\
& =\left(N_{0} V_{0}^{H}\right)\left(\varphi_{0}+\Phi a\right)-N_{0} f=N_{0}\left[V_{0}^{H}\left(\varphi_{0}+\Phi a\right)-f\right] ;
\end{aligned}
$$

so, by Theorem 4(ii),

$$
V_{0}^{H}\left(\varphi_{0}+\Phi a\right)-f=F k
$$


for some constant $k \in \mathscr{M}_{3 \times 1}$ that depends on $a$. In view of Theorem 1(ix), this equality can be rewritten as

$$
V_{0}^{H} \varphi_{0}-f=F k-\left(V_{0} \Phi+F H p \Phi\right) a=F[k-(H+\mathscr{C}) a]=F l,
$$

where $l \in \mathscr{M}_{3 \times 1}$ is constant and independent of $a$.

Let $b=(H+\mathscr{C})^{-1} l$, which is well defined since we assumed that $\operatorname{det}(H+\mathscr{C}) \neq 0$. Then

$$
\begin{aligned}
V_{0}^{H}\left(\varphi_{0}-\Phi b\right)-f & =V_{0}^{H} \varphi_{0}-\left(V_{0} \Phi+F H p \Phi\right) b-f \\
& =V_{0}^{H} \varphi_{0}-F(H+\mathscr{C})(H+\mathscr{C})^{-1} l-f=0 ;
\end{aligned}
$$

consequently, $\varphi=\varphi_{0}-\Phi b \in C^{0, \alpha}(\partial S)$ is a solution of (17).

The solution is unique since the difference $\varphi=\varphi_{1}-\varphi_{2}$ of two solutions $\varphi_{1}$ and $\varphi_{2}$ satisfies $V_{0}^{H} \varphi=0$, which, by Theorem 2, implies that $\varphi=0$.

Arbitrariness of the representation. To establish the arbitrariness of the representation of the solution of $\left(\mathrm{D}^{+}\right)$as a modified single layer potential, we need the following assertion.

THEOREM 6. If $V_{0} \varphi=F k$, where $\varphi \in \mathscr{M}_{2 \times 1}$ and $k \in \mathscr{M}_{3 \times 1}$ is a constant vector, then $\varphi=\Phi \gamma$ and $k=\mathscr{C} \gamma$ for some constant $\gamma \in \mathscr{M}_{3 \times 1}$.

Proof. Let $\Psi \in \mathscr{M}_{2 \times 3}$ be such that $\Psi^{(\beta)}=\Phi^{(\beta)}, \beta=1,2, \Psi^{(3)}=\varphi$, and suppose that $\operatorname{det}(p \Psi) \neq 0$. Then, by Theorem $1(\mathrm{ix})$,

$$
\begin{aligned}
& V_{0} \Psi=F \mathscr{D}, \quad \mathscr{D}^{(\beta)}=\mathscr{C}^{(\beta)}, \quad \mathscr{D}^{(3)}=k, \\
& p\left(\Psi(p \Psi)^{-1}\right)=(p \Psi)(p \Psi)^{-1}=E_{3} .
\end{aligned}
$$

Again by Theorem 1(ix), we must have

$$
\Psi(p \Psi)^{-1}=\Phi, \quad \mathscr{D}(p \Psi)^{-1}=\mathscr{C} ;
$$

therefore, $\Psi=\Phi(p \Psi)$ and $\mathscr{D}=\mathscr{C}(p \Psi)$, from which

$$
\varphi=\Psi^{(3)}=\Phi(p \Psi)^{(3)}=\Phi \gamma, \quad k=\mathscr{D}^{(3)}=\mathscr{C}(p \Psi)^{(3)}=\mathscr{C} \gamma,
$$

with $\gamma=p \varphi$.

If $\operatorname{det}(p \Psi)=0$, then there is a constant nonzero $c \in \mathscr{M}_{3 \times 1}$ such that $(p \Psi) c=0$. Let $\Lambda \in \mathscr{M}_{3 \times 3}$ be such that $\Lambda^{(\beta)}=0, \beta=1,2, \Lambda^{(3)}=c$. Then, by (19),

$$
\begin{aligned}
V_{0}(\Phi+\Psi \Lambda) & =F \mathscr{C}+F \mathscr{D} \Lambda=F(\mathscr{C}+\mathscr{D} \Lambda), \\
p(\Phi+\Psi \Lambda) & =E_{3}+p(\Psi \Lambda)=E_{3} .
\end{aligned}
$$

As above, this means that

$$
\Phi+\Psi \Lambda=\Phi, \quad \mathscr{C}+\mathscr{D} \Lambda=\mathscr{C} ;
$$

consequently, $\Psi \Lambda=0$ and $\mathscr{D} \Lambda=0$. From this it follows that $\Psi c=0$ and $\mathscr{D} c=0$, which can also be written as

$$
c_{1} \Phi^{(1)}+c_{2} \Phi^{(2)}+c_{3} \varphi=0, \quad c_{1} \mathscr{C}^{(1)}+c_{2} \mathscr{C}^{(2)}+c_{3} k=0 .
$$


It is clear that $c_{3} \neq 0$, since, by Theorem $1(\mathrm{ix})$, the $\Phi^{(i)}$ are linearly independent. Setting $\gamma=-c_{3}^{-1}\left(c_{1}, c_{2}, 0\right)^{\mathrm{T}}$, we now see that $\varphi=\Phi \gamma$. and $k=\mathscr{C} \gamma$. The theorem is proved.

Suppose now that $u=V^{H_{1}} \varphi^{(1)}=V^{H_{2}} \varphi^{(2)}, H_{1} \neq H_{2}, H_{\beta}^{\mathrm{T}}=H_{\beta}, \operatorname{det}\left(H_{\beta}+\mathscr{C}\right) \neq 0$, $\beta=1,2$. Then

$$
V_{0}\left(\varphi^{(1)}-\varphi^{(2)}\right)=F\left(H_{2} p \varphi^{(2)}-H_{1} p \varphi^{(1)}\right) \text {; }
$$

so, by Theorem 6 ,

$$
\varphi^{(1)}-\varphi^{(2)}=\Phi \gamma, \quad H_{2} p \varphi^{(2)}-H_{1} p \varphi^{(1)}=\mathscr{C} \gamma
$$

for some constant $\gamma \in \mathscr{M}_{3 \times 1}$. Hence,

$$
p \varphi^{(1)}-p \varphi^{(2)}=(p \Phi) \gamma=\gamma,
$$

which, in view of $(20)$, yields

$$
\mathscr{C}\left(p \varphi^{(1)}-p \varphi^{(2)}\right)=\mathscr{C} \gamma=H_{2} p \varphi^{(2)}-H_{1} p \varphi^{(1)}
$$

or

$$
\left(H_{1}+\mathscr{C}\right) p \varphi^{(1)}=\left(H_{2}+\mathscr{C}\right) p \varphi^{(2)} .
$$

From this it follows that

$$
p \varphi^{(2)}=\left(H_{2}+\mathscr{C}\right)^{-1}\left(H_{1}+\mathscr{C}\right) p \varphi^{(1)} .
$$

Replacing in (21), we find that

$$
\gamma=\left[E_{3}-\left(H_{2}+\mathscr{C}\right)^{-1}\left(H_{1}+\mathscr{C}\right)\right] p \varphi^{(1)}
$$

therefore, by (20),

$$
\varphi^{(2)}=\varphi^{(1)}-\Phi\left[E_{3}-\left(H_{2}+\mathscr{C}\right)^{-1}\left(H_{1}+\mathscr{C}\right)\right] p \varphi_{1} .
$$

This formula indicates how any other representation can be generated from a specific one corresponding to a given matrix $H_{1}$.

The exterior Dirichlet problem. Here we want to find $u \in C^{2}\left(S^{-}\right) \cap C^{1}\left(\bar{S}^{-}\right)$such that

$$
\begin{aligned}
A u & =0 \text { in } S^{-}, \\
u & =f \text { on } \partial S, \\
u(r, \theta) & =M^{\infty}(r, \theta) q+\sigma^{\mathscr{A}^{*}} \text { as } r=|x| \rightarrow \infty,
\end{aligned}
$$

where $f \in C^{1, \alpha}(\partial S), q \in \mathscr{M}_{3 \times 1}$ is a given constant vector, and $\sigma^{\mathscr{A}^{*}} \in \mathscr{A}^{*}$. The last equation represents the most general far-field pattern for an analytic solution of (1) in $S^{-}$[5]. Clearly, $\left(\mathrm{D}^{-}\right)$has at most one solution, since the difference $u$ of two solutions satisfies $\left.u\right|_{\partial S}=0$ and $u \in \mathscr{A}^{*}$; therefore, by Theorem 1 (viii), $u=0$ in $S^{-}$.

It is already known from the solution of $\left(\mathrm{D}^{+}\right)$that there is a $u \in \mathscr{M}_{3 \times 1}$ that satisfies the first two equations of $\left(\mathrm{D}^{-}\right)$. This is $u=V^{H} \varphi$, which has the far-field pattern

$$
u=M^{\infty} p \varphi+F H p \varphi+\sigma^{\mathscr{A}}=M^{\infty} p \varphi+\sigma^{\mathscr{A}^{*}} \text { as } r \rightarrow \infty .
$$


To obtain the required behaviour at infinity, we seek the solution of $\left(\mathrm{D}^{-}\right)$as

$$
u=V^{L} \psi, \quad \psi=\varphi+\Phi k,
$$

where $L \in \mathscr{M}_{3 \times 3}$ and $k \in \mathscr{M}_{3 \times 1}$ are constant matrices with $L^{\mathrm{T}}=L$.

As $|x| \rightarrow \infty$,

$$
\begin{aligned}
V^{L} \psi & =V^{L} \varphi+\left(V^{L} \Phi\right) k \\
& =M^{\infty} p \varphi+F L p \varphi+M^{\infty}(p \Phi) k+F L(p \Phi) k+\sigma^{\mathscr{A}} \\
& =M^{\infty}(p \varphi+k)+\sigma^{\mathscr{A}^{*}} .
\end{aligned}
$$

Thus, we must have

$$
k=q-p \varphi .
$$

We can write

$$
V^{L} \varphi=V \varphi+F L p \varphi=V \varphi+F H p \varphi+F(L-H) p \varphi=V^{H} \varphi+F(L-H) p \varphi ;
$$

so on $\partial S$

$$
\begin{aligned}
V_{0}^{L} \psi & =V_{0}^{L} \varphi+\left(V_{0}^{L} \Phi\right)(q-p \varphi) \\
& =V_{0}^{H} \varphi+F(L-H) p \varphi+\left(V_{0} \Phi+F L p \Phi\right)(q-p \varphi) \\
& =f+F[(L-H) p \varphi+(L+\mathscr{C})(q-p \varphi)] .
\end{aligned}
$$

Suppose that $q \neq 0$. Then $V_{0}^{L} \psi=f$ if

$$
L q=H p \varphi+\mathscr{C}(p \varphi-q)=(H+\mathscr{C}) p \varphi-\mathscr{C} q .
$$

Obviously, there is arbitrariness in $L$, but the representation of $u$ as $V^{L} \psi$ reduces to the same expression regardless of any admissible choice of $L$ :

$$
\begin{aligned}
u & =V^{L} \psi=V^{L}(\varphi+\Phi k)=V^{L}[\varphi+\Phi(q-p \varphi)] \\
& =V[\varphi+\Phi(q-p \varphi)]+F L p[\varphi+\Phi(q-p \varphi)]=V[\varphi+\Phi(q-p \varphi)]+F L q \\
& =V[\varphi+\Phi(q-p \varphi)]+F[(H+\mathscr{C}) p \varphi-\mathscr{C} q] .
\end{aligned}
$$

If $q=0$, then, by (22) and (23), $k=-p \varphi$ and

$$
V_{0}^{L} \psi=f-F(H+\mathscr{C}) p \varphi .
$$

Consequently, $L$ plays no role in the solution, so we may take, for example, $L=H$. Also, we need to add a correction term to the solution, which now becomes

$$
u=V^{H} \psi+F(H+\mathscr{C}) p \varphi=V(\varphi-\Phi p \varphi)+F(H+\mathscr{C}) p \varphi .
$$

Evidently, in this case $\left.u\right|_{\partial S}=f$ and $u \in \mathscr{A}^{*}$, as required.

\section{REFERENCES}

[1] F. J. Rizzo, An integral equation approach to boundary value problems of classical elastostatics, Quart. Appl. Math. 25, 83-95 (1967)

[2] C. Constanda, On the direct method of boundary integral equations in plane elasticity, Quart. J. Mech. Appl. Math. 47, 261-268 (1994) 
[3] G. C. Hsiao and W. L. Wendland, On a boundary integral method for some exterior problems in elasticity, Trudy Tbiliss. Univ. Mat. Mekh. Astronom. 18, 31-60 (1985)

[4] C. Constanda, The boundary integral equation method in plane elasticity, Proc. Amer. Math. Soc. 123, 3385-3396 (1995)

[5] N. I. Muskhelishvili, Some basic problems in the mathematical theory of elasticity, 3rd ed., P. Noordhoff, Groningen, 1949 